Sharif, A.M., Elliman, T., and Badii, A. (2004). "Integrating the IS with the Enterprise : key EAI research challenges". Forthcoming in Journal of Enterprise Information Management, 17 (1 / 2).

\title{
Integrating the IS with the Enterprise: key EAI research challenges
}

\author{
Amir M. Sharif \\ Information Systems Evaluation \& Integration Network Group (ISE-ING) \\ Department of Information Systems \& Computing \\ Brunel University \\ United Kingdom \\ Tony Elliman \\ Information Systems Evaluation \& Integration Network Group (ISE-ING) \\ Department of Information Systems \& Computing \\ Brunel University \\ United Kingdom \\ Email: tony.elliman@brunel.ac.uk
}

\author{
Atta Badii \\ P3ie-EnCkompass \\ Information Systems Department \\ University College Northampton \\ United Kingdom \\ Email: Atta.Badii@Northampton.ac.uk
}

\begin{abstract}
Enterprise Application Integration (EAI) technologies provide the means to integrate strategic business solutions within and across the component parts of organisational information system infrastructures. The continuing development of both digitally integrated business models, through various eCommerce and eBusiness initiatives, has meant that the importance of EAI within enterprise IS, has increased significantly. Noting that EAI incurs not only technological but stakeholder-level commitments, this paper outlines the product of a sustained investigation into key challenges within Enterprise IS and EAI, and provides a framework for future research and investigation into this emerging and evolving area.
\end{abstract}

Keywords: Enterprise Systems, EAI, Information Systems research 
Sharif, A.M., Elliman, T., and Badii, A. (2004). "Integrating the IS with the Enterprise : key EAI research challenges". Forthcoming in Journal of Enterprise Information Management, 17 (1 / 2).

\section{Introduction}

The increasing deployment of enterprise applications alongside legacy systems, has meant that companies are being compelled to adopt Information Systems (IS) infrastructures that connect applications, data and information together. The approach that provides a solution to this is Enterprise Application Integration (EAI). Notions of integration across application and organisation have been around for many years (Elliman and Sanger, 1991) before they achieved the status of a defined branch of IS technology and in a fluid business world the need to integrate extant systems will continually arise. EAI is not a transient phenomenon because the systems it generates today will be the disintegrated legacy systems of the next decade. This paper outlines some of the key challenges within Enterprise systems implementation and EAI, which have arisen as a result of ongoing debate within a themed network consisting of both practitioners and IS researchers.

Undoubtedly, the continued development and progression of computing and IS has led many organisations and indeed economies, to transform themselves. The usage and implementation of information technology, has allowed many organisations to also institute information systems that encode and embody the business strategy completely (Davenport, 1998; Doukidis et al., 1998). The advent of resource and schedule planning systems, such as Enterprise Resource Planning (ERP), has meant that the sharing of common data and information across the enterprise is now an inherent necessity for many organisations (Eck and Marchetti, 2000; Al-Mashari and Zairi, 2000). As such, it can be said that although EAI is not necessarily a novel approach to integrating IS components, it is a tool which has proved to be useful when the integration of many disparate systems needs to be carried out (Linthicum, 1999). Organisations with large and complex IS infrastructures, typically those who have experienced the benefits and limitations of mergers and acquisitions activity, are the target of such approaches (Hasselbring, 2000; Klasell and Dudgeon, 1998). Since the aim of any EAI approach is to provide technologies, processes and procedures for smoothing information flow, it is not difficult to see that the main beneficiaries from this field have been software vendors and professional service organisations.

Prompted by the continuing, but unresolved, debate about IS failures and the need the need for effective pre and post implementation evaluation techniques Brunel University proposed the formation of the Network for IS Evaluation (N-ISE). This, with the aid of EPSRC funding, brought together academics from eight Universities and professional colleagues from six industrial or public bodies. The remit for the network was to promote national debate, to extend the research base, and to establish a frame of reference for discussing IS evaluation issues.

At its very first meeting in December 2000 the network members identified EAI as a particular area of concern. EAI is backroom technology - it is supportive of the IS function within an organisation but not directly visible to the general staff or its clients and stakeholders. Existing work in IS planning and evaluation (e.g. Coakes and Elliman, 1999; Clarke et al, 2000) had already shown the need to address any IS project in its wider organisational context. Thus IS integration within the enterprise should also encompass more than just the technological aspects. Not only should EAI and enterprise IS include concepts of information integration in terms of integration across data, information and processes, but it should also be considered that integration across many levels of the IS organisation itself, needs to be achieved.

In order to explore these concepts further, N-ISE established a working group, led by the authors. In this paper we report on the work of that group and our conclusions. 
Sharif, A.M., Elliman, T., and Badii, A. (2004). "Integrating the IS with the Enterprise : key EAI research challenges". Forthcoming in Journal of Enterprise Information Management, 17 (1 / 2).

\section{Investigating themes within EAI: a research agenda}

The major research theme within the group, was to understand and delineate how the concept of EAI, its technology, methodology and implementation, would be able to provide benefit and value to an organisation's IS function and processes. This was in order to understand, and ultimately evaluate, the relative impact of EAI on an organisation's business, its IT infrastructure and crucially, on people and work processes. In order to confirm the existence of these factors, concepts of IS evaluation from both industry and academia (Willcocks, 1994), were used as guiding principles behind understanding both organisational and lifecycle aspects of EAI. Hence, the industry-academe research focus was to aggregate experiences and research relating to:

- Required systems infrastructures (technical and organisational) needed to mediate between various forms of IS;

- Identifying IS components and / or functions within an organization;

- Assessing the relative impact of application integration approaches upon a model of an ISdependent organization.

Figure 1, shows the overall scope of the group's debate in terms of technology, process and transformation. Upon formation, the key issues that the group identified were related to the capability, delivery, evaluation and overall impact of EAI. Furthermore, through identifying key technologies (core technology definitions), processes (a mapping of lifecycle models) and transformation characteristics (aspects of change), the group has highlighted core issues within the area. These areas of interest are now discussed in further detail.

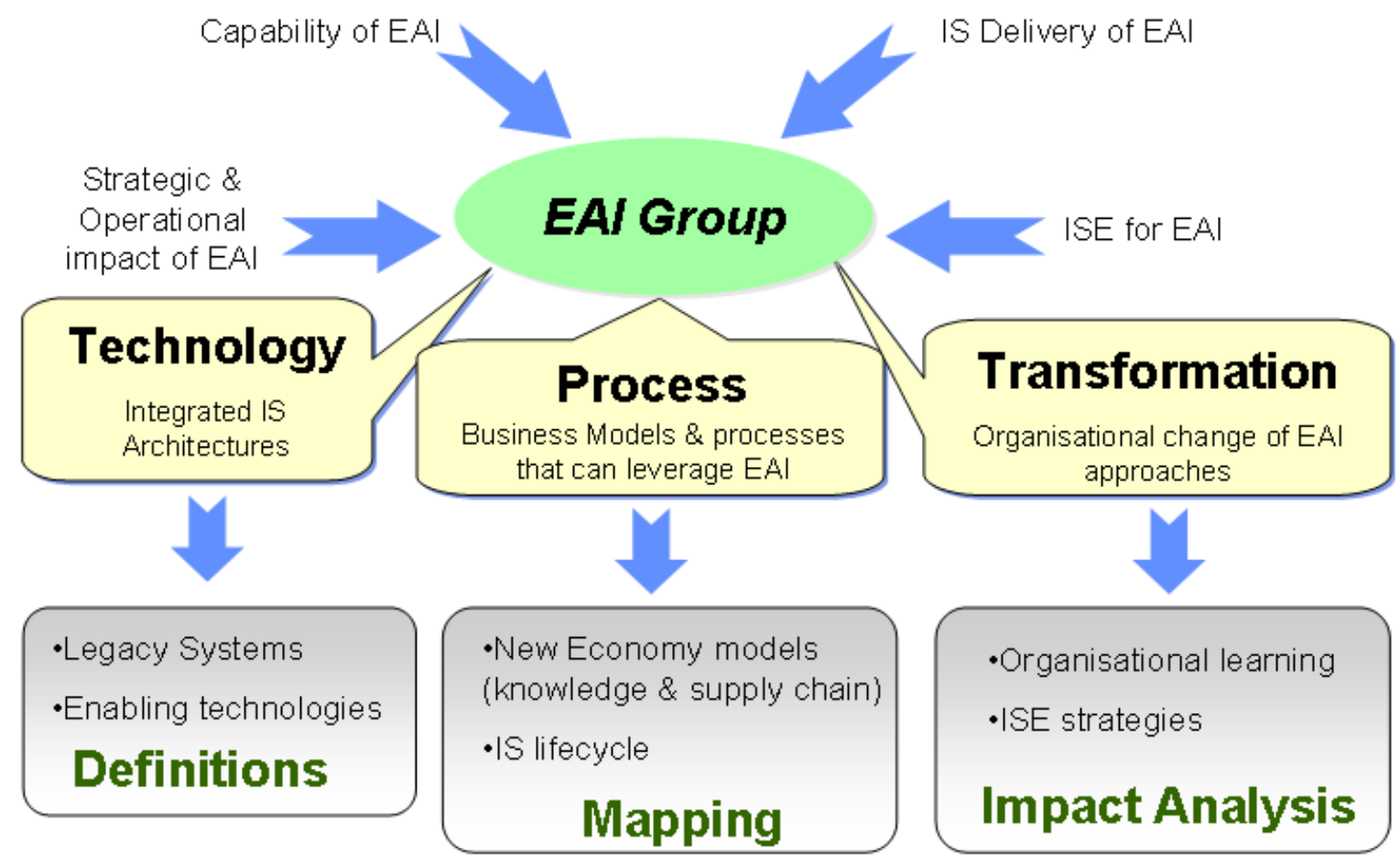

Figure 1. Research focus of the N-ISE EAI workgroup 
Sharif, A.M., Elliman, T., and Badii, A. (2004). "Integrating the IS with the Enterprise : key EAI research challenges". Forthcoming in Journal of Enterprise Information Management, 17 (1 / 2).

Through a series of workshops, presentations and working papers, members of the working group highlighted a number of key challenges that were difficult to answer, by both practitioner and academic alike. At the very top of the list were problems with the definition of EAI itself. Since the concept of EAI is to integrate information and processes via technology, what should be the ultimate benchmark and capability profile of such a technology? The organisational context also appears to be crucial to understanding the nature of EAI. The needs and requirements of tradition business structures with concrete physical and manpower assets are quite different from those of a wholly virtual organisation or an organisation based upon notions such as an extended supply chain.

It follows from this that the evaluation of EAI projects will also be context dependant. Not just that, as with all evaluation, the context drives the evaluation, but that the evaluation techniques must match the EAI technologies with their characteristic definitions; understanding the significance and context of EAI successes and failures; providing a taxonomy of IS evaluation approaches appropriate to EAI; and the impact of EAI upon organisational work \& culture (i.e. the fit of soft systems approaches). These challenges led to the generation of 5 key research questions:

- How does integration fit into the continuum of electronic business and commerce?

- What is the current and future fit of EAI with other forms of IS (MIS, DSS, EIS)?

- How does the "life" of the enterprise impact upon the infrastructure that supports it?

- Who are the 'Victims' \& 'Beneficiaries' of enterprises that employ EAI solutions?

- How does EAI relate to notions of knowledge management and collaboration?

As can be seen from this list, few of the research questions focus on the purely technological aspects. Indeed, many of the questions have inherent people-focussed components relating to them. By providing a grouping of these key challenges and research questions, a framework for executing practitioner-academe research in EAI, was formulated, as shown in Figure 2. 
Sharif, A.M., Elliman, T., and Badii, A. (2004). "Integrating the IS with the Enterprise : key EAI research challenges". Forthcoming in Journal of Enterprise Information Management, 17 (1 / 2).

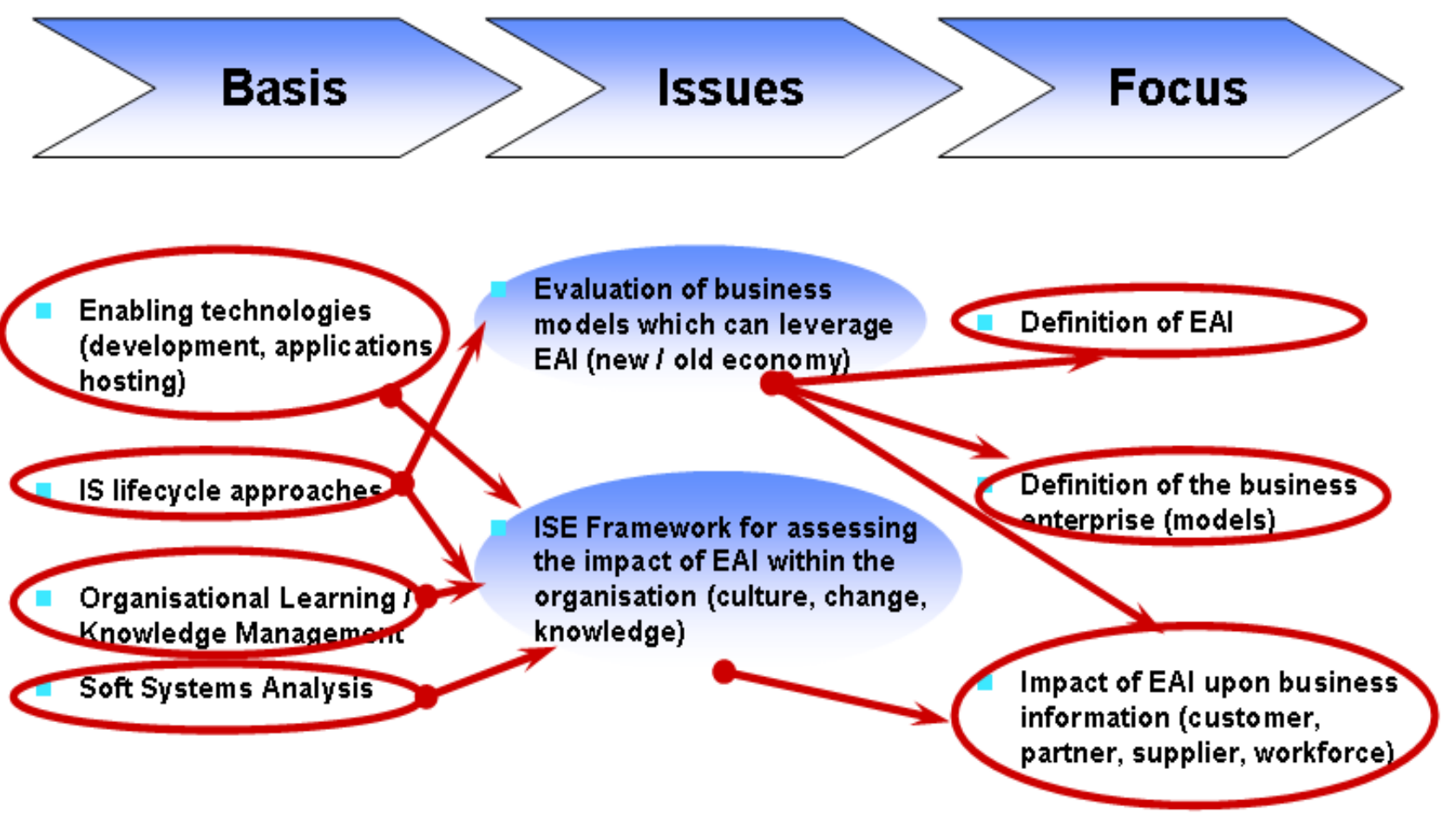

Figure 2. Research themes and questions within EAI

This framework of research themes and questions within EAI highlights two key issues within EAI research today. The first of these, is grounded in the current research on the topic and which has been investigated by many practitioners and academics alike. The technological aspect and impact of integrative technology, is almost trivial, in the sense that selection and implementation of EAI for most business models is an imperative. Online retailers such as Amazon would not be as successful as they are today, if it were not for the consistency of information across their procure-to-pay supply chain. Existing retailers are faced with either building a stand-alone online business or using EAI methodology to integrate the online business with their current systems and Earl (2001) has argued that, in the long run, isolated development is a less mature and less effective business strategy." Hence, the evaluation of business models which can leverage such integration technologies, is highly dependent upon the choice and existence of the particular organisation IS lifecycle and process which is chosen. By understanding the context of such business models, a better definition of EAI, in terms of the scope of integration, could possibly be achieved, along with the impact of integration upon knowledge-based work.

The second issue raised was to do with the potential use of IS evaluation methods in order to assess the impact of EAI upon the organisational culture. EAI research to-date is almost exclusively focussed on the technology and other reviews (Johannesson et al. 2000) maintain this research direction. Broader research to assess the human impact of EAI is also essential. This essentially, stakeholderbased view of EAI will require a multidisciplinary approach with the ability to see beyond the technological challenge. This must still address the attributes of the underlying technology but also involve aspects of the IS lifecycle required in order to implement and deploy such a solution; the capacity and capability for the organisation to learn and adopt integrative information and knowledge 
Sharif, A.M., Elliman, T., and Badii, A. (2004). "Integrating the IS with the Enterprise : key EAI research challenges". Forthcoming in Journal of Enterprise Information Management, 17 (1 / 2).

management practices; and an analysis of the underlying decision workflow, using approaches such as soft systems analysis. Because of the wide remit of such a research agenda in this regard, the ultimate focus would shift from inter-enterprise components (internal IT/IS, lifecycles, business models, stakeholders), to intra-enterprise components (customers, suppliers, partners and the like).

The remit of the workgroup, therefore centred on these core issues involving not only the technology but also the human-centric aspect, within the overall EAI debate. In order to make these concepts progressive and realisable, the following section describes a framework that encompasses these ideas.

\section{A generic, EAI impact framework}

Given the research aims and issues highlighted previously, the workgroup decided to focus on defining the largely sociological aspects of the research model. Hence, initial work was carried out in order to provide a model for describing "end-to-end" IS-mediated integration and evaluation, in order to maintain and enhance an organisation's business model (Badii and Sharif, 2003 ; Badii and Sharif, 2002). The framework realised, showed the convergence of technology, information content, and collaborative knowledge exists within many modern organisations (Galliers \& Newell, 2000), as shown in Figure 3. This model is based upon earlier work which has focussed on the concept of negotiability-centric information systems architectures such as C-Assure (Cultural Accommodation with Sensitised Systems for Usability Relationships Evaluation (Badii 1999, Badii 2000a-e). The guiding principle behind the C-Assure approach, has been to include psycho-social and psychophysiologically-grounded patterns of in relation to enterprise IS (i.e. system adoption and avoidance, stakeholder interrelationships, and other interactions with IS). Thus the information integration framework presented, provides the integration of not only technology, but also those aspects of decision-workflow which engender the realisation of enterprise integration. 
Sharif, A.M., Elliman, T., and Badii, A. (2004). "Integrating the IS with the Enterprise : key EAI research challenges". Forthcoming in Journal of Enterprise Information Management, 17 (1 / 2).

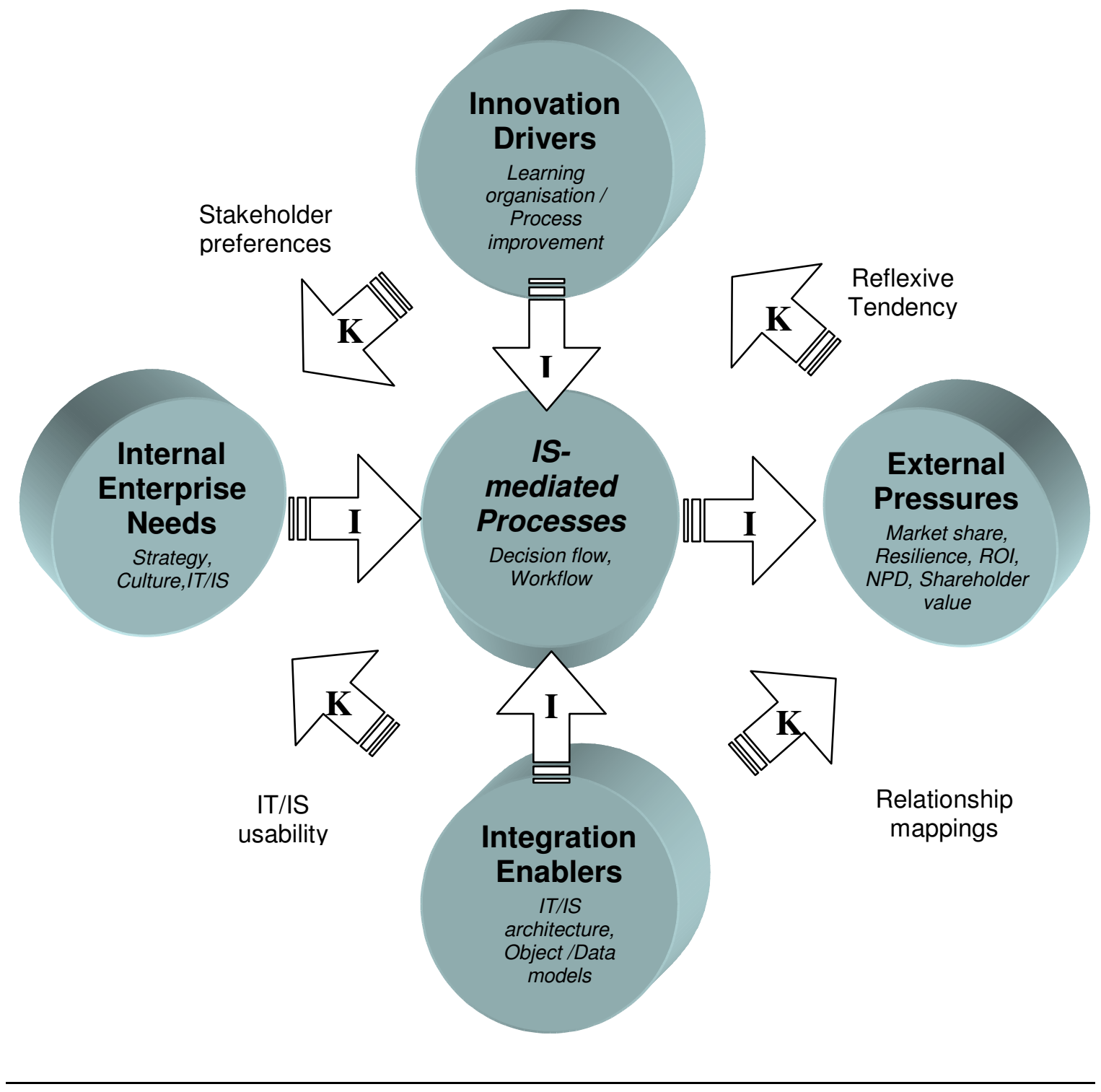

Figure 3. An integrated Enterprise Information Integration and Knowledge framework

As such, Figure 3 shows both the direct IT/IS as well as indirect, reflexive factors which tend to enable integration of information and sharing of knowledge, across the enterprise. Although it is conventional to talk of evaluation in terms of costs and benefits these words often imply too close a focus on financial measures. Therefore our model is based on measurable benefits, dis-benefits, sideeffects and affects within the enterprise environment. These are shown in the diagram as the feedback between external competitive pressures and internal, enterprise requirements and based upon information and knowledge dependencies. This is in terms of both innovation drivers and integration enablers (for example, organisation learning and EAI interfaces). 
Sharif, A.M., Elliman, T., and Badii, A. (2004). "Integrating the IS with the Enterprise : key EAI research challenges". Forthcoming in Journal of Enterprise Information Management, 17 (1 / 2).

Extending this model further, based upon the previously published value-chain model for enterprise integration (Badii \& Sharif, 2002), an impact framework which encompasses the key themes and challenges outlined previously is now shown in Figure 4.

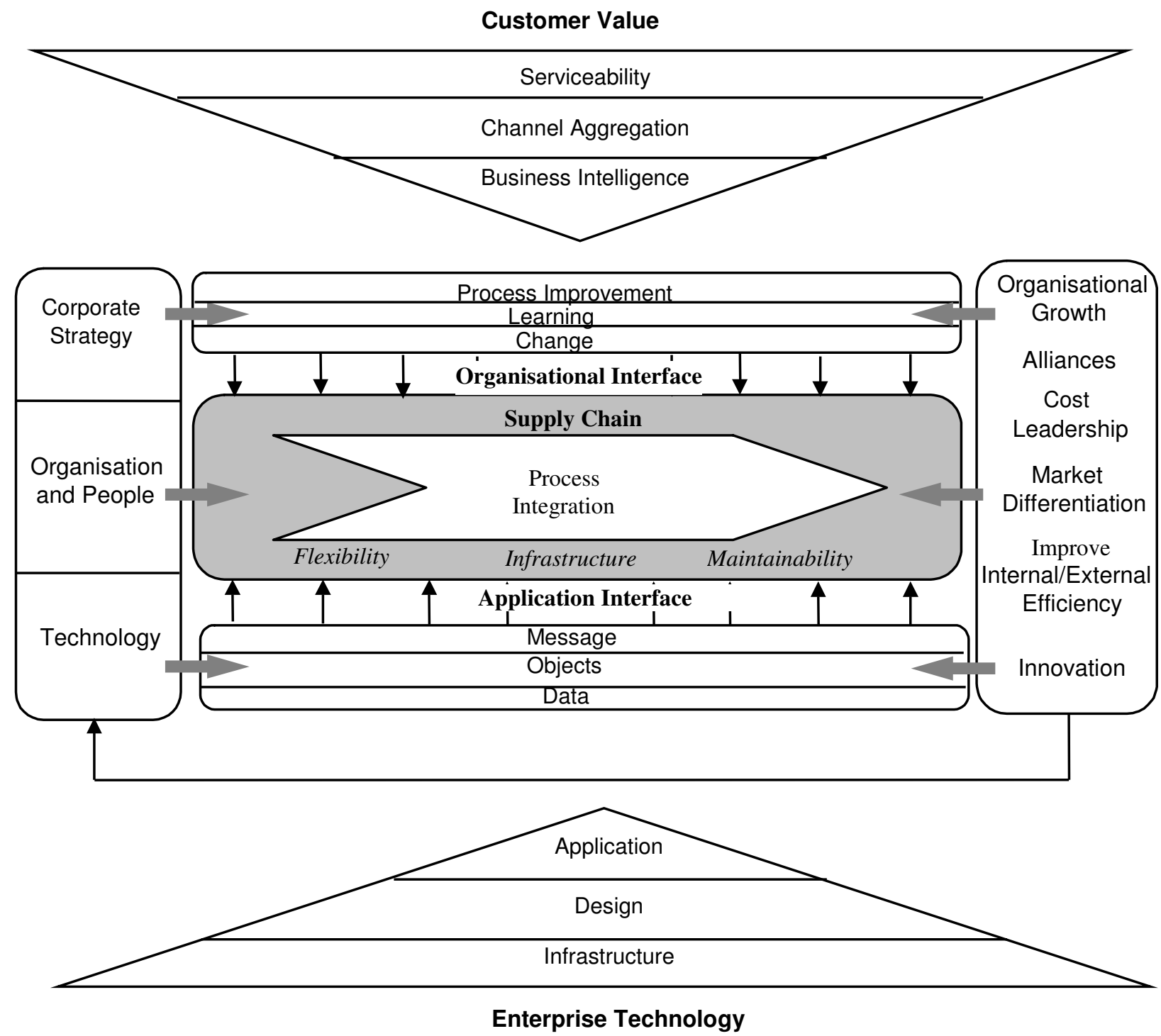

Figure 4. EAI Impact framework

This framework, which is sector-independent, aggregates both the inter and intra-enterprise enablers and drivers from both the identified research themes and the knowledge and integration framework in Figure 3. This diagram essentially outlines a type of value-chain analysis of the core components of a modern organisation. Again, the left hand side of the diagram associates factors involved with the internal enterprise needs, upheld via integration enablers and supplanted by IS-mediated process (from above and below the centre of the diagram respectively). Whilst the right hand side of the 
Sharif, A.M., Elliman, T., and Badii, A. (2004). "Integrating the IS with the Enterprise : key EAI research challenges". Forthcoming in Journal of Enterprise Information Management, 17 (1 / 2).

diagram, is associated with those external pressures and innovation drivers which impinge upon the organisation's internal supply chain characteristics (i.e. flexibility, infrastructure, maintainability). The organisational interface to the external world, such as the customer in this case, must involve a mapping between internal process and change factors, and external service and market requirement capabilities. That is to say, that any organisation wishing to leverage its internally integrated architecture, must similarly extend the results of any such integration outwards, to give business benefit to the customer.

\section{Conclusion}

EAI is a backroom technology, which is often perceived primarily as a productivity tool for IS professionals, but its role and impact can't be evaluated outside of the specific business context the target systems support. EAI is also a broad concept covering a wide range of EAI software products, processes and methods, and research is need to classify the business problems and contexts that are appropriate for each of these offerings.

The real impact of EAI is a second or third hand effect on the organisation's core business. Thus research and the development of appropriate pre and post implementation evaluation techniques must be human focussed and look to identify the impact on working practices. The measurement techniques need to capture benefits, dis-benefits and side-effects of the project if they are to be effective.

Finally research needs to address the long-term impact of EAI practices on the culture of an organisation. Since EAI will always be needed to address the demand for change in a company's business we must ask research questions like "How does EAI relate to notions of knowledge sharing and collaboration within and between organisations?"

\section{Acknowledgements}

The authors wish to gratefully acknowledge the support from the Network for IS Evaluation (NISE) Research Programme within which this work was carried out. NISE was established at Brunel University Department of Information Systems and Computing, in December 2000, under a research grant from the UK Engineering and Physical Sciences Research Council (EPSRC: GR/R08025/01).

\section{References}

Badii, A. (1999). The BSIRM_MB SmartProject Best Practice Framework, in Proceedings of the special Research and Technology Development Project Management Workshop, Sponsored by the European Directorate General of the Fifth European Research Programme, DGX111, EUFP5 IST, Brussels October 1999, published November 1999.

Badii, A. (2000a). Summary Proceedings of UKAIS (Midlands) Research Seminar Series, UKAIS Newsletter June 2000. 
Sharif, A.M., Elliman, T., and Badii, A. (2004). "Integrating the IS with the Enterprise : key EAI research challenges". Forthcoming in Journal of Enterprise Information Management, 17 (1 / 2).

Badii, A. (2000b). Design of architectures for forum management and re-negotiability in virtual environments: Proceedings of the 1st EnCKompass International Research Network Workshop, University College Northampton, July 2000.

Badii, A. (2000c). Man-machine mutuality and semiotics, evolving web pages as re-adaptive social stereotypes with attitude, Proceedings of the 2nd EnCKompass International Research Network Workshop Trinity College Dublin September 2000.

Badii, A., and Sharif, A.M. (2002). Enterprise Challenges: Information Management, Knowledge Integration \& Deployment. In Proc. International Conference on Systems Thinking in Management 2002 (ICSTM 2002), Salford University, UK.

Badii, A., and Sharif, A.M. (2003). "Integrating Information and Knowledge for Enterprise Innovation”. Logistics Information Management, 16 (2) : 145 - 155.

Clarke, S., Elliman, T. and Lehaney, B. (2000) Re-engineering an Information System: A Case Study in Risk Reduction, International Journal of Flexible Manufacturing Systems, 12 (4) : 305320.

Coakes, E. and Elliman, T. (1999) The Role of Stakeholders in Managing Change, Communications of the Association for Information Systems, 2 (July) article 4 (available at: http://cais.aisnet.org/articles/default.asp?vol=2\&art=4).

Davenport, T. (1998). Putting the Enterprise into the Enterprise System. Harvard Business Review Jul-Aug 1998, pp. 121-131.

Doukidis, G., Poulymenakou, A., Terpsidis, J., Themistocleous, M. and Miliotis, P. (1998). 'Electronic Commerce: Challenging Issues and the Impact on Employment', European Union, DG5, Brussels, Belgium.

Earl, M. J. (2001). Evolving the E-Business. Centre for the Networked Economy, London Business School, London, NW1 4SA: Working Paper CNE WP02/2001

Elliman, T. and Sanger, C. (1991). Open Systems for Europe, Chapman-Hall.

Eck, J. and Marchetti, N. (2000). Combing e-Commerce \& EAI, EAI Journal, January 2000, pp. 4243

Galliers, R.D., and, Newell, S. (2000). Back to the future: from knowledge management to data management. London School of Economics, Dept. of Information Systems, Working Paper Series, Number 92.

Hasselbring, W. (2000). Information System Integration, Communications of the ACM, 43 (6) : 33 38.

Johannesson, P., Wangler, B. and Jayaweera, P. (2000). Application and Process Integration Concepts, Issues, and Research Directions. In Information Systems Engineering Symposium: State of the Art and Research Themes, Stockholm, Sweden, June 5-6, 2000, Springer-Verlag, London: 159-169.

Klasell, T., and Dudgeon, S. (1998). Enterprise Application Integration. Dain Rauscher Wessels, NY : New York.

Linthicum, D. (1999). Enterprise Application Integration. Addison-Wesley, Boston : MA.

Willcocks, L. (1994). Information Management - The Evaluation of Information Systems Investments. Chapman and Hall, London. 